\title{
Tiotropium Versus Placebo for Inadequately Controlled Asthma: A Meta-Analysis
}

\author{
Jing-wei Tian MD, Jin-wu Chen MD, Rui Chen PhD, and Xin Chen PhD
}

\begin{abstract}
OBJECTIVE: This meta-analysis was performed to evaluate the efficacy and safety of the addition of tiotropium to standard treatment regimens for inadequately controlled asthma. METHODS: A systematic search was made of PubMed, EMBASE, MEDLINE, and CENTRAL databases, and ClinicalTrials.gov, and a hand search of leading respiratory journals. Randomized, double-blind clinical trials on the treatment of inadequately controlled asthma for $\geq 4$ weeks with the addition of tiotropium, compared with placebo, were reviewed. Studies were pooled to odds ratio (OR) and weighted mean differences (WMDs), with 95\% CI. RESULTS: Six trials met the inclusion criteria. The addition of tiotropium, compared with placebo, significantly improved all spirometric indices, including morning and evening peak expiratory flow (WMD $20.59 \mathrm{~L} / \mathrm{min}, 95 \%$ CI 15.36-25.81 L/ min, $P<.001$; and WMD $24.95 \mathrm{~L} / \mathrm{min}, 95 \%$ CI 19.22-30.69 L/min, $P<.001$, respectively), trough and peak $\mathrm{FEV}_{1}$ (WMD $0.13 \mathrm{~L}, 95 \%$ CI 0.09-0.18 L, $P<.001$; and WMD $0.10 \mathrm{~L}, 95 \% \mathrm{CI}$ $0.06-0.14 \mathrm{~L}, P<.001$, respectively), the area under the curve of the first $3 \mathrm{~h}$ of $\mathrm{FEV}_{1}$ (WMD $0.13 \mathrm{~L}$, 95\% CI 0.08-0.18 L, $P<.001$ ), trough and peak FVC (WMD $0.1 \mathrm{~L}, 95 \%$ CI $0.05-0.15 \mathrm{~L}, P<.001$; and WMD $0.08 \mathrm{~L}, 95 \%$ CI $0.04-0.13 \mathrm{~L}, P<.001$, respectively), the area under the curve of the first $3 \mathrm{~h}$ of FVC (WMD $0.11 \mathrm{~L}, 95 \%$ CI $0.06-0.15 \mathrm{~L}, P<.001$ ). The mean change in the 7-point Asthma Control Questionnaire score (WMD $-0.12,95 \%$ CI -0.21 to $-0.03, P=.01$ ) was markedly lower in tiotropium group, but not clinically important. There were no significant differences in Asthma Quality of Life Questionnaire score (WMD 0.09, 95\% CI -0.01 to $0.20, P=.09$ ), night awakenings (WMD 0.00, 95\% CI -0.05 to $0.05, P=.99$ ) or rescue medication use (WMD $-0.18,95 \% \mathrm{CI}-0.36$ to $0.00, P=.06)$. No significant increase was noticed in adverse events in the tiotropium group (OR $0.80,95 \%$ CI $0.62-1.03, P=.08)$. CONCLUSIONS: The addition of tiotropium to standard treatment regimens has significantly improved lung function without increasing adverse events in patients with inadequately controlled asthma. Long-term trials are required to assess the effects of the addition of tiotropium on asthma exacerbations and mortality. Key words: asthma; inadequately controlled asthma; meta-analysis; anticholinergics; tiotropium. [Respir Care 2014;59(5):654-666.

(C) 2014 Daedalus Enterprises]
\end{abstract}

\section{Introduction}

Asthma is a chronic respiratory disease characterized by reversible airway obstruction that is secondary to airway

\footnotetext{
Drs Tian and Rui Chen are affiliated with the Department of Respiratory Diseases, and Dr Jin-wu Chen is affiliated with the Medical Examination Center, Sun Yat-sen Memorial Hospital, Sun Yat-Sen University, Guangzhou 510120, China. Dr Xin Chen is affiliated with the Department of Respiratory Diseases, Zhujiang Hospital, Southern Medical University, Guangzhou 510280, China.
}

The authors have disclosed no conflicts of interest. inflammation and excessive smooth muscle contraction. ${ }^{1}$ A great proportion of patients with asthma experience recurring symptoms and exacerbations, even after the administration of high doses of inhaled corticosteroids combined with a long-acting $\beta_{2}$ agonists. The Global Initiative for Asthma guidelines recommend the addition of another

\footnotetext{
Correspondence: Rui Chen PhD, Department of Respiratory Diseases, Sun Yat-Sen Memorial Hospital, Sun Yat-Sen University, 107 Yan Jiang Road, Guangzhou 510120, China. E-mail: gzchenrui@163.com.
}

DOI: $10.4187 /$ respcare. 02703 
medication, such as antileukotrienes, theophyllines, antiIgE, and immunosuppressants (eg, systemic corticosteroids or cyclosporine), to achieve optimal asthma control. ${ }^{2} \mathrm{Nev}$ ertheless, many patients do not achieve symptom control with current options. Furthermore, there are also concerns about the safety of regular use of high-dose long-acting $\beta_{2}$ agonists and inhaled corticosteroids in patients with asthma. Adding a second bronchodilator with a different mechanism of action into the treatment of inadequately controlled asthma might be a new available way to address the problem.

The parasympathetic nervous system is an important neural pathway that controls airway smooth muscle by muscarinic receptors. Stimulation of the parasympathetic nerve can result in bronchoconstriction, bronchial vasodilatation, and mucus secretion. Moreover, recent investigations revealed that the non-neuronal cholinergic system was widely expressed in epithelial cells, eosinophils, submucosal glands, smooth muscle cells, and a variety of immune cells including lymphocytes, macrophages, and mast cells in the airway, suggesting that non-neuronal cholinergic signals played an important role in the pathophysiology of asthma. ${ }^{3}$ Therefore, it seems favorable to add an anticholinergic agent to block cholinergic signals in the treatment of asthma. Previous studies found no long-term benefits of short-acting anticholinergic agents in patients with persistent asthma. ${ }^{4,5}$ Tiotropium bromide is an anticholinergic agent with long-lasting action that is characterized by a slow dissociation from acetylcholine M1 and M3 receptors. ${ }^{6,7}$ Current COPD treatment guidelines recommend tiotropium as the first-choice long-acting bronchodilator for maintenance therapy in patients with moderate or severe COPD because of its effectiveness, safety, and convenient once-daily dosing. ${ }^{8}$ However, little has been known about its efficacy in patients with asthma. In animal models of allergic asthma it was shown that tiotropium inhibited airway inflammation and reduced airway remodeling. ${ }^{910}$ Recently, beneficial effects of tiotropium maintenance dosing in patients with asthma have been reported in clinical study. Peters et al ${ }^{11}$ demonstrated that the addition of tiotropium improved symptoms and lung function in patients with mild-to-moderate asthma that had been poorly controlled with only low-dose inhaled corticosteroids, and its effects were found not to be inferior to those of salmeterol. In addition, Bateman et al ${ }^{12}$ reported that tiotropium was not inferior to salmeterol in maintaining improved lung function in B16-Arg/Arg patients with asthma and that the addition of tiotropium to treatment with a high-dose inhaled corticosteroid plus a long-acting $\beta_{2}$ agonist improved lung function in patients with poorly controlled severe asthma. ${ }^{16}$

The aim of the present meta-analysis was to evaluate the efficacy and safety of tiotropium versus placebo in asthmatic patients whose symptoms were inadequately con-

\section{QUICK LOOK}

\section{Current knowledge}

Asthma is a common obstructive airway disease typically managed with inhaled bronchodilators and corticosteroids. The benefits of combining two long-acting bronchodilators with different modes of action have been reported in patients with COPD.

\section{What this paper contributes to our knowledge}

This meta-analysis suggests that the addition of tiotropium to the treatment of inadequately controlled asthma, compared with placebo, may improve lung function without an increase in adverse events.

trolled with standard treatment regimens (ie, inhaled corticosteroids with or without long-acting $\beta_{2}$ agonists).

\section{Methods}

\section{Data Sources}

We searched PubMed, EMBASE, MEDLINE, and CENTRAL databases and ClinicalTrials.gov for trials published from January 1980 to December 2012 using the following search terms: "tiotropium OR Ba 679 BR OR Spiriva AND asthma." These searches were supplemented by hand searching of leading respiratory journals and conference abstracts. All publications and abstracts in the English language were considered. Moreover, a further search in April 2013 did not identify additional trials that fulfilled our search criteria.

\section{Study Selection}

The inclusion criteria of trials were as follows: (1) double-blind randomized controlled trials (RCTs) on tiotropium compared with placebo; (2) duration of at least 4 weeks; (3) $\geq 12$ y of age; (4) patients with symptomatic asthma even after treatment with an inhaled corticosteroid or an inhaled corticosteroid plus long-acting $\beta_{2}$ agonist; (5) a history of asthma without other lung diseases; and (6) a modified Jadad score of $\geq 4$ points.

\section{Quality Assessment}

The methodological quality of each study was assessed by the Modified Jadad Scale (7 points), ${ }^{13}$ which scores trials according to randomization, concealment of allocation, double blinding, withdrawals, and dropouts. Studies with a score of $\geq 4$ points were included. 


\section{Data Extraction}

Data extraction was based on reported statistics (means, $\mathrm{SD}$, and $\mathrm{SE}$ ) for the intention-to-treat population. Two authors (J-wT and $\mathrm{J}-\mathrm{wC}$ ) independently extracted data from the selected studies. If disagreement arose, all the authors conferred until a consensus was reached. Authors of a publication were contacted if only its abstract was available or data were missing. Primary outcomes were changes from baseline in morning and evening peak expiratory flow (PEF). Secondary outcomes included changes from baseline in peak and trough $\mathrm{FEV}_{1}$, peak and trough $\mathrm{FVC}$, the areas under the curve of the first $3 \mathrm{~h}$ of $\mathrm{FEV}_{1}$ and $\mathrm{FVC}$, nighttime awakenings, rescue bronchodilator use, 7-point Asthma Control Questionnaire (ACQ-7) score, Asthma Quality of Life Questionnaire (AQLQ) score, and adverse events. The ACQ is a questionnaire consisting of a 7-point scale ranging from 0 (no impairment) to 6 (maximum impairment), with a minimal clinically important difference of 0.5 units.

\section{Statistical Analysis}

RevMan (Review Manager, version 5.2, The Nordic Cochrane Centre, The Cochrane Collaboration, Copenhagen, Denmark) was used to analyze all collected data. Fixed-effects odds ratios (OR) for dichotomous outcomes and the weighted mean difference (WMD) for continuous outcomes, with corresponding 95\% CIs, were calculated for individual trials. Trials were pooled using a fixedeffects OR or WMD as appropriate. Heterogeneity was tested with a $P$ value $<.1$, which is considered statistically significant. The inconsistency $\left(\mathrm{I}^{2}\right)$ test was also calculated to efficiently test heterogeneity, with values of $25 \%, 50 \%$, and $75 \%$ considered to represent low, moderate, and high heterogeneity, respectively. The differences between patients receiving tiotropium and those receiving placebo were pooled using a fixed-effects model when there was no evidence of significant heterogeneity in the analysis; if significant heterogeneity was found a random-effects model was used. ${ }^{14}$ Publication bias was examined using funnel plots. ${ }^{15}$

\section{Results}

\section{Search Results}

The progress of searching and selecting trials is presented in Figure 1. Of the 42 English-language articles that were screened, we excluded 37 that were either not relevant or had incomplete data. To reduce heterogeneity across different trials, we selected only those comparing tiotropium (5 $\mu \mathrm{g}$ once a day, with Respimat inhaler) with placebo at both baseline and at the end of the treatment pe- riod. Five articles involving 1,648 participants, including 6 RCTs (3 parallel RCTs and 3 crossover RCTs) that met our inclusion criteria were selected for the present metaanalysis. Characteristics of the trials we included are shown in Table 1 and Table 2. All data adopted in the present study had been published openly either on a web site (http:// ClinicalTrials.gov) or in journals.

\section{Primary Outcome}

Change in Morning and Evening PEF. The 6 trials included took morning and evening PEF values as end points. The results of each study showed significant improvements in morning and evening PEF in subjects treated with tiotropium. The overall analysis showed statistically significant improvements in morning PEF (WMD $20.25 \mathrm{~L} /$ $\min , 95 \%$ CI $15.36-25.81 \mathrm{~L} / \mathrm{min}, P<.001)$ and in evening PEF (WMD $24.95 \mathrm{~L} / \mathrm{min}, 95 \%$ CI 19.22-30.69 L/ min, $P<.001$ ) in the tiotropium group (Fig. 2).

\section{Secondary Outcomes}

Change in $\mathbf{F E V}_{\mathbf{1}}$. Five trials reported peak and trough $\mathrm{FEV}_{1}$, and 4 trials reported the area under the curve of the first $3 \mathrm{~h}$ of $\mathrm{FEV}_{1}$. The results of each study showed significantly greater improvements in peak and trough $\mathrm{FEV}_{1}$ in subjects treated with tiotropium than in those treated with placebo. The pooled analysis (1,260 participants) showed statistically significant improvements in peak FEV (WMD $0.13 \mathrm{~L}, 95 \%$ CI $0.09-0.18 \mathrm{~L}, P<.001$ ) and in

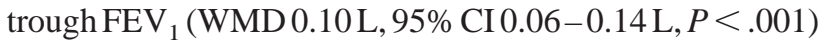
in the tiotropium group. Three trials showed obvious improvements in the area under the curve of the first $3 \mathrm{~h}$ of $\mathrm{FEV}_{1}$ in the tiotropium group, although one study showed no significant differences between the two groups. The pooled analysis showed a statistically significant improvement in the area under the curve of the first $3 \mathrm{~h}$ of $\mathrm{FEV}_{1}$ (WMD $0.13 \mathrm{~L}, 95 \%$ CI $0.08-0.18 \mathrm{~L}, P<.001$ ) in the tiotropium group. Nevertheless, improvement in $\mathrm{FEV}_{1}$ was not nearly the minimum clinically important difference of $230 \mathrm{~mL}$ in asthma ${ }^{20}$ (Fig. 3).

Change in FVC. Five included trials reported the FVC. Although no obvious improvements in peak FVC, trough $\mathrm{FVC}$, and area under the curve of the first $3 \mathrm{~h}$ of FVC were observed in any single study, the cumulative analysis showed a statistically significant improvement, respectively, in peak FVC (WMD $0.10 \mathrm{~L}, 95 \%$ CI $0.06-0.14 \mathrm{~L}$, $P<.001$ ), trough FVC (WMD $0.08 \mathrm{~L}, 95 \%$ CI $0.04-$ $0.13 \mathrm{~L}, P<.001)$, and area under the curve of the first $3 \mathrm{~h}$ of FVC (WMD $0.11 \mathrm{~L}, 95 \%$ CI $0.06-0.15 \mathrm{~L}, P<.001$ ) in tiotropium group (Fig. 4). 


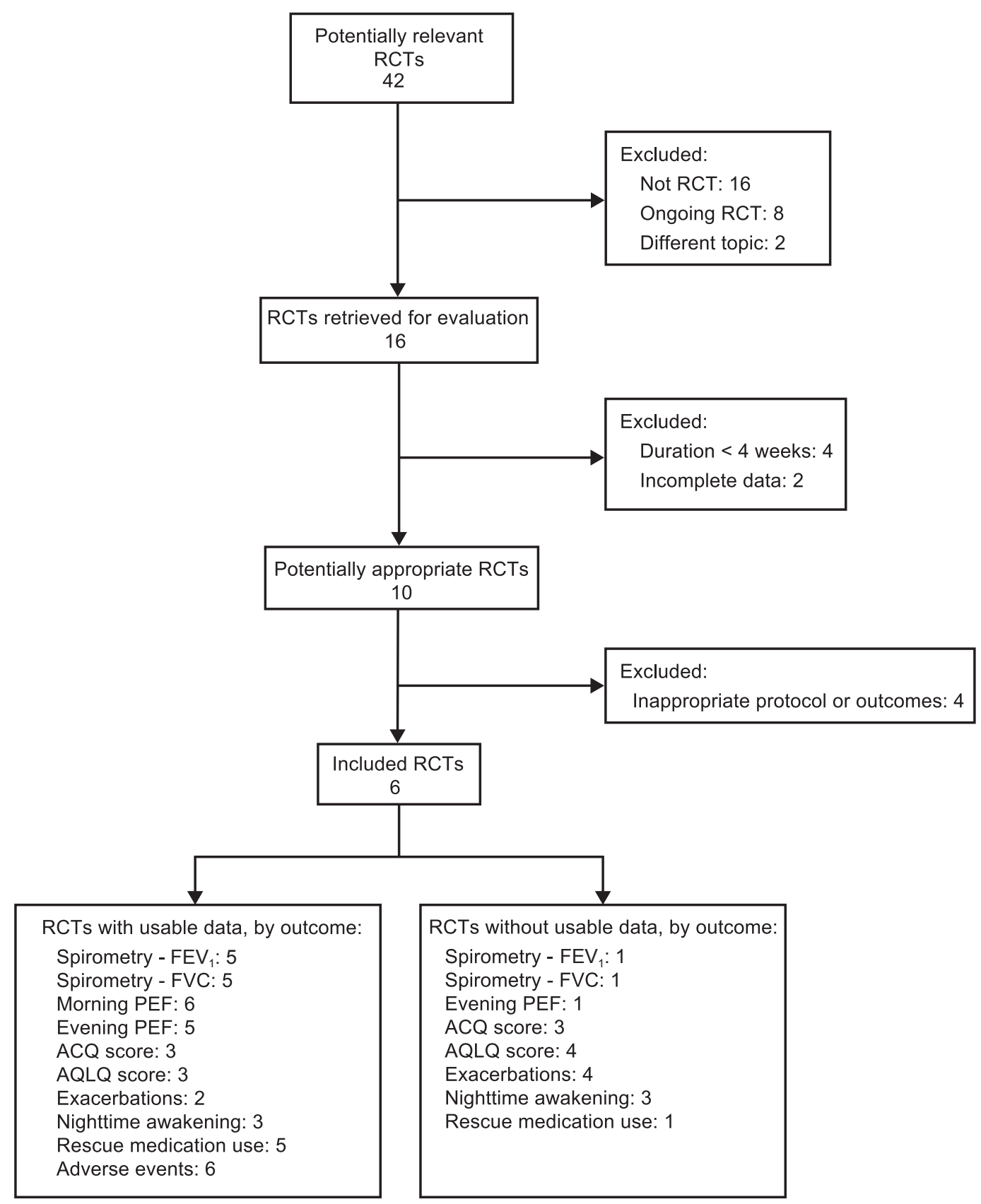

Fig. 1. Flow chart for identification of relevant studies. RCT = randomized controlled trials, $A C Q=$ Asthma Control Questionnaire, $A Q L Q=$ Asthma Quality of Life Questionnaire, PEF = peak expiratory flow.

Asthma Control. Of the trials included, 3 reported score of the ACQ-7. The score was statistically lower with tiotropium than with placebo (WMD $-0.12,95 \%$ CI -0.21 to $-0.03, P=.01)$. However, the improvement in ACQ-7 score did not achieve the minimum clinically important difference of 0.5 units in asthma.

Night Awakenings. Three trials showed data for the mean number of night awakenings during the last week of treatment. The cumulative analysis showed no statistical dif- ferences between patients receiving tiotropium and those receiving placebo (WMD $0.00,95 \% \mathrm{CI}-0.05$ to 0.05 ; $\left.\mathrm{I}^{2}=0 \%, P=.99\right)$ (Fig. 5).

Rescue Medication Use. The mean number of puffs of rescue medication during the whole day in the last week of treatment was reported in 5 trials. Although the pooled analysis showed a dropping trend in patients receiving tiotropium compared with those receiving placebo 
Tiotropium Versus Placebo for Inadequately Controlled Asthma

Table 1. Characteristics of Participants of Included Studies

\begin{tabular}{|c|c|c|c|c|c|c|}
\hline Reference & $\begin{array}{l}\text { Participants, } \\
\quad n\end{array}$ & $\begin{array}{c}\text { Age, } y, \\
\text { Mean } \pm \text { SD }\end{array}$ & $\begin{array}{c}\text { Female/ } \\
\text { Male }\end{array}$ & $\begin{array}{c}\text { Treatment } \\
\text { Duration, } \\
\text { wk }\end{array}$ & $\begin{array}{l}\text { Inclusion } \\
\text { Criteria }\end{array}$ & $\begin{array}{l}\text { Exclusion } \\
\text { Criteria }\end{array}$ \\
\hline Bateman et $\mathrm{al}^{12}$ & 388 & $43.3 \pm 12.6$ & $240 / 148$ & 16 & $\begin{array}{l}\text { Age } 18-67 \mathrm{y} \text {; homozygous for arginine at } \\
\text { the } 16 \text { th amino acid position of the } \beta_{2} \\
\text { adrenergic receptor (B16 Arg/Arg); } \\
\text { have a documented history of asthma; } \\
\text { moderate persistent asthma }\end{array}$ & $\begin{array}{l}\text { Significant diseases other than asthma; } \\
\text { myocardial infarction } \leq 6 \text { months; } \\
\text { hospitalization for cardiac failure } \\
\leq 1 \text { year; life-threatening cardiac } \\
\text { arrhythmia; resection, radiation therapy, } \\
\text { or chemotherapy for }<5 \text { y; COPD; } \\
\text { active tuberculosis; pulmonary } \\
\text { rehabilitation program of } \leq 6 \text { weeks }\end{array}$ \\
\hline \multicolumn{7}{|l|}{ Kerstjens et al ${ }^{16}$} \\
\hline Trial 2 & 453 & $52.5 \pm 12.1$ & $262 / 191$ & 48 & $\begin{array}{l}\text { Age } 18-75 \text { y old; at least a } 5 \text {-y history of } \\
\text { asthma; treated severe persistent } \\
\text { asthma }\end{array}$ & $\begin{array}{l}\text { Abnormal hematology findings; } \\
\text { myocardial infarction } \leq 6 \text { months; } \\
\text { hospitalization for cardiac failure; life- } \\
\text { threatening cardiac arrhythmia; active } \\
\text { tuberculosis; resection, radiation } \\
\text { therapy or chemotherapy for }<5 \text { y; } \\
\text { lung diseases other than asthma } \\
\text { (eg, COPD); asthma exacerbation or } \\
\text { respiratory tract infection for }<4 \\
\text { weeks }\end{array}$ \\
\hline Trial 1 & 459 & $53.4 \pm 12.6$ & $289 / 170$ & 48 & $\begin{array}{l}\text { Age } 18-75 \text { y old; at least a } 5 \text {-y history of } \\
\text { asthma; treated severe persistent } \\
\text { asthma }\end{array}$ & $\begin{array}{l}\text { Abnormal hematology findings; } \\
\text { myocardial infarction } \leq 6 \text { months; } \\
\text { hospitalization for cardiac failure; life- } \\
\text { threatening cardiac arrhythmia; active } \\
\text { tuberculosis; resection, radiation } \\
\text { therapy, or chemotherapy for }<5 \mathrm{y} \text {; } \\
\text { lung diseases other than asthma (eg, } \\
\text { COPD); asthma exacerbation or } \\
\text { respiratory tract infection for }<4 \\
\text { weeks }\end{array}$ \\
\hline NCT01122680 & 105 & $14.0 \pm 1.5$ & $38 / 67$ & 4 & $\begin{array}{l}\text { Age } 12-17 \text { y old; at least a } 3 \text {-month } \\
\text { history of asthma; moderate persistent } \\
\text { asthma; } \mathrm{FEV}_{1} \geq 60 \% \text { and } \leq 90 \% \\
\text { predicted normal; bronchodilator } \\
\text { reversibility* }\end{array}$ & $\begin{array}{l}\text { Congenital or acquired heart disease; life- } \\
\text { threatening cardiac arrhythmia; } \\
\text { resection, radiation therapy or } \\
\text { chemotherapy for }<5 \text { y; lung diseases } \\
\text { other than asthma; narrow-angle } \\
\text { glaucoma; renal impairment }\end{array}$ \\
\hline NCT01233284 ${ }^{18}$ & 149 & $49.3 \pm 13.3$ & $82 / 67$ & 4 & $\begin{array}{l}\text { Age } 18-75 \mathrm{y} \text {; at least a } 3 \text {-month history } \\
\text { of asthma; bronchodilator reversibility* }\end{array}$ & $\begin{array}{l}\text { Abnormal hematology findings; } \\
\text { myocardial infarction } \leq 6 \text { months; } \\
\text { hospitalization for cardiac failure; life- } \\
\text { threatening cardiac arrhythmia; active } \\
\text { tuberculosis; resection, radiation } \\
\text { therapy, or chemotherapy for }<5 \mathrm{y} \text {; } \\
\text { lung diseases other than asthma; } \\
\text { moderate-to-severe renal impairment; } \\
\text { narrow-angle glaucoma }\end{array}$ \\
\hline NCT01 152450 & 94 & $44.3 \pm 13.2$ & $55 / 39$ & 4 & $\begin{array}{l}\text { Age } 18-75 \text { y old; at least a } 3 \text {-month } \\
\text { history of asthma; moderate persistent } \\
\text { asthma; } \mathrm{FEV}_{1} \geq 60 \% \text { and } \leq 90 \% \\
\text { predicted normal; bronchodilator } \\
\text { reversibility* }\end{array}$ & $\begin{array}{l}\text { Abnormal hematology findings; } \\
\text { myocardial infarction } \leq 6 \text { months; } \\
\text { hospitalization for cardiac failure; life- } \\
\text { threatening cardiac arrhythmia; active } \\
\text { tuberculosis; resection, radiation } \\
\text { therapy, or chemotherapy for }<5 \mathrm{y} \text {; } \\
\text { lung diseases other than asthma (eg, } \\
\text { COPD); pregnant or nursing women }\end{array}$ \\
\hline
\end{tabular}

\footnotetext{
* Bronchodilator reversibility is defined as an increase in $\mathrm{FEV}_{1}$ of $\geq 12 \%$ and $\geq 200 \mathrm{~mL} 15$ min after administration of $400 \mu \mathrm{g}$ of salbutamol.
} 
Tiotropium Versus Placebo for Inadequately Controlled Asthma

Table 2. Studies Included in the Present Analysis

\begin{tabular}{|c|c|c|c|c|c|c|}
\hline Reference & $\begin{array}{l}\text { Control } \\
\text { Design }\end{array}$ & End Point & Treatment Groups & Basic Drugs & $\begin{array}{l}\text { Tiotropium } \\
\text { Inhaler }\end{array}$ & $\begin{array}{l}\text { Jadad } \\
\text { Scale }\end{array}$ \\
\hline Bateman et a ${ }^{12}$ & Parallel & $\begin{array}{l}\text { Primary outcome measures: } \\
\text { morning PEF } \\
\text { Secondary outcome measures: } \\
\text { morning and evening PEF, } \\
\text { morning and evening FEV } \\
\text { asthma symptoms, Mini-AQLQ } \\
\text { score, blood pressure, pulse rate }\end{array}$ & $\begin{array}{l}\text { Tiotropium } 5 \mu \mathrm{g} \\
\text { once daily; } \\
\text { salmeterol } \\
50 \mu \mathrm{g} \text { twice daily }\end{array}$ & Inhaled corticosteroid & Respimat & 6 \\
\hline \multicolumn{7}{|l|}{ Kerstjens et al ${ }^{16}$} \\
\hline Trial 2 & Parallel & $\begin{array}{l}\text { Primary outcome measures: peak } \\
\mathrm{FEV}_{1} \text {, trough } \mathrm{FEV}_{1} \text { and time to } \\
\text { first severe asthma exacerbation } \\
\text { Secondary outcome measures: area } \\
\text { under the curve of the first } 3 \mathrm{~h} \\
\text { of } \mathrm{FEV}_{1}, \mathrm{FVC}, \mathrm{PEF} \text {, asthma } \\
\text { exacerbations, hospitalizations } \\
\text { for exacerbations, AQLQ score, } \\
\text { ACQ score, asthma symptom- } \\
\text { free days, rescue medication use }\end{array}$ & $\begin{array}{l}\text { Tiotropium } 5 \mu \mathrm{g} \\
\text { once daily }\end{array}$ & $\begin{array}{l}\text { Inhaled corticosteroid } \\
\text { and long-acting } \beta_{2} \\
\text { agonist }\end{array}$ & Respimat & 6 \\
\hline Trial 1 & Parallel & $\begin{array}{l}\text { Primary outcome measures: peak } \\
\mathrm{FEV}_{1} \text {, trough } \mathrm{FEV}_{1} \text {, and time to } \\
\text { first severe asthma exacerbation } \\
\text { Secondary outcome measures: area } \\
\text { under the curve of the first } 3 \mathrm{~h} \\
\text { of } \mathrm{FEV}_{1}, \mathrm{FVC}, \mathrm{PEF} \text {, asthma } \\
\text { exacerbations, hospitalizations } \\
\text { for exacerbations, AQLQ score, } \\
\text { ACQ score, asthma symptom- } \\
\text { free days, rescue medication use }\end{array}$ & $\begin{array}{l}\text { Tiotropium } 5 \mu \mathrm{g} \\
\text { once daily }\end{array}$ & $\begin{array}{l}\text { Inhaled corticosteroid } \\
\text { and long-acting } \beta_{2} \\
\text { agonist }\end{array}$ & Respimat & 6 \\
\hline NCT01122680 ${ }^{17}$ & Crossover & $\begin{array}{l}\text { Primary outcome measures: peak } \\
\text { FEV }_{1} \\
\text { Secondary outcome measures: } \\
\text { trough and area under the curve } \\
\text { of the first } 3 \text { h of } \mathrm{FEV}_{1}, \mathrm{FVC}, \\
\text { PEF, rescue medication use, } \\
\text { ACQ score, nighttime } \\
\text { awakenings }\end{array}$ & $\begin{array}{l}\text { Tiotropium } 1.25 \mu \mathrm{g} \\
\text { once daily; } \\
\text { tiotropium } 2.5 \mu \mathrm{g} \\
\text { once daily; } \\
\text { tiotropium } 5 \mu \mathrm{g} \\
\text { once daily }\end{array}$ & Inhaled corticosteroid & Respimat & 4 \\
\hline NCT0123328418 & Crossover & $\begin{array}{l}\text { Primary outcome measures: peak } \\
\text { FEV }_{1} \\
\text { Secondary outcome measures: } \\
\text { trough and area under the curve } \\
\text { of the first } 3 \mathrm{~h} \text { of } \mathrm{FEV}_{1}, \mathrm{FVC}, \\
\text { PEF, rescue medication use, } \\
\text { nighttime awakenings }\end{array}$ & $\begin{array}{l}\text { Tiotropium } 1.25 \mu \mathrm{g} \\
\text { once daily; } \\
\text { tiotropium } 2.5 \mu \mathrm{g} \\
\text { once daily; } \\
\text { tiotropium } 5 \mu \mathrm{g} \\
\text { once daily }\end{array}$ & $\begin{array}{l}\text { Inhaled corticosteroid } \\
\text { alone or with long- } \\
\text { acting } \beta_{2} \text { agonist } \\
\text { or short-acting } \beta_{2} \\
\text { agonist }\end{array}$ & Respimat & 4 \\
\hline NCT01152450 ${ }^{19}$ & Crossover & $\begin{array}{l}\text { Primary outcome measures: area } \\
\text { under the curve of the first } 3 \mathrm{~h} \\
\text { of } \mathrm{FEV}_{1} \\
\text { Secondary outcome measures: } \\
\text { PEF, } \mathrm{FEV}_{1}, \mathrm{FVC} \text {, rescue } \\
\text { medication use, nighttime } \\
\text { awakenings }\end{array}$ & $\begin{array}{l}\text { Tiotropium } 2.5 \mu \mathrm{g} \\
\text { twice daily; } \\
\text { tiotropium } 5 \mu \mathrm{g} \\
\text { once daily. }\end{array}$ & $\begin{array}{l}\text { Inhaled corticosteroid } \\
\text { alone or with long- } \\
\text { acting } \beta_{2} \text { agonist } \\
\text { or short-acting } \beta_{2} \\
\text { agonist }\end{array}$ & Respimat & 4 \\
\hline \multicolumn{7}{|c|}{$\begin{array}{l}\mathrm{PEF}=\text { peak expiratory flow } \\
\mathrm{AQLQ}=\text { Asthma Quality of Life Questionnaire } \\
\mathrm{ACQ}=\text { Asthma Control Questionnaire }\end{array}$} \\
\hline
\end{tabular}


A.

\begin{tabular}{|c|c|c|c|c|c|c|c|c|c|c|}
\hline \multirow{2}{*}{ Study or Subgroup } & \multicolumn{3}{|c|}{ Tiotropium } & \multicolumn{3}{|c|}{ Placebo } & \multirow{2}{*}{ Weight } & \multirow{2}{*}{$\begin{array}{l}\text { Mean Difference IV, } \\
\text { Random, 95\% CI }\end{array}$} & \multirow{2}{*}{\multicolumn{2}{|c|}{$\begin{array}{l}\text { Mean Difference IV, } \\
\text { Random, 95\% CI }\end{array}$}} \\
\hline & Mean & SD & Total & Mean & SD & Total & & & & \\
\hline Bateman 2011 & -3.93 & 55.132 & 128 & -24.63 & 54.057 & 125 & $15.1 \%$ & $20.70[7.25,34.15]$ & & . \\
\hline Kerstjens 2012 trial-2 & 17.396 & 60.787 & 216 & -3.258 & 60.226 & 232 & $21.7 \%$ & $20.65[9.44,31.87]$ & & $\rightarrow$ \\
\hline Kerstjens 2012-trial-1 & 15.297 & 55.452 & 237 & -6.996 & 55.933 & 222 & $26.3 \%$ & $22.29[12.10,32.49]$ & & \\
\hline NCT01122680 & 20.491 & 53.605 & 79 & 7.267 & 52.563 & 73 & $9.6 \%$ & $13.22[-3.66,30.11]$ & & -7 \\
\hline NCT01152450 & 24.31 & 55.572 & 88 & 1.953 & 55.939 & 91 & $10.2 \%$ & $22.36[6.02,38.69]$ & & $<$ \\
\hline NCT01233284 & 25.241 & 54.708 & 144 & 4.395 & 54.494 & 142 & $17.1 \%$ & $20.85[8.19,33.50]$ & $\begin{array}{rl}1 & 1 \\
-20 & -10 \\
-20 & 0\end{array}$ & 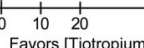 \\
\hline Total $(95 \% \mathrm{CI})$ & & & 892 & & & 885 & $100.0 \%$ & $20.59[15.36,25.81]$ & & \\
\hline
\end{tabular}

Heterogeneity: $\mathrm{Tau}^{2}=0.00 ; \mathrm{Chi}^{2}=0.89, \mathrm{df}=5(P=.97) ; \mathrm{I}^{2}=0 \%$

Test for overall effect: $\mathrm{Z}=7.72(P<.001)$

B.

\begin{tabular}{|c|c|c|c|c|c|c|c|c|c|}
\hline \multirow{2}{*}{ Study or Subgroup } & \multicolumn{3}{|c|}{ Tiotropium } & \multicolumn{3}{|c|}{ Placebo } & \multirow{2}{*}{ Weight } & \multirow{2}{*}{$\begin{array}{l}\text { Mean Difference IV, } \\
\text { Random, 95\% CI }\end{array}$} & \multirow{2}{*}{$\begin{array}{c}\text { Mean Difference IV, } \\
\text { Random, 95\% CI }\end{array}$} \\
\hline & Mean & SD & Total & Mean & SD & Total & & & \\
\hline Kerstjens 2012 trial-2 & 25.158 & 64.622 & 216 & -7.295 & 63.79 & 232 & $23.2 \%$ & $32.45[20.55,44.35]$ & \\
\hline Kerstjens 2012-trial-1 & 19.402 & 56.499 & 237 & -3.865 & 57.453 & 222 & $30.2 \%$ & $23.27[12.83,33.70]$ & \\
\hline NCT01122680 & 16.565 & 53.063 & 79 & -0.552 & 52.101 & 73 & $11.8 \%$ & $17.12[0.39,33.84]$ & 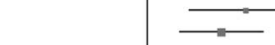 \\
\hline NCT01152450 & 27.096 & 55.953 & 89 & -1.56 & 56.578 & 91 & $12.2 \%$ & $28.66[12.22,45.10]$ & \\
\hline NCT01233284 & 25.414 & 52.176 & 144 & 3.833 & 51.991 & 142 & $22.6 \%$ & $21.58[9.51,33.65]$ & $\begin{array}{llll}10 & 0 & 10 & 20\end{array}$ \\
\hline Total $(95 \% \mathrm{CI})$ & & & 765 & & & 760 & $100.0 \%$ & $24.95[19.22,30.69]$ & Favors [Placebo] $\quad$ Favors [Tiotrop \\
\hline
\end{tabular}

Heterogeneity: $\mathrm{Tau}^{2}=0.00 ; \mathrm{Chi}^{2}=2.96, \mathrm{df}=4(P=.56) ; \mathrm{I}^{2}=0 \%$

Test for overall effect: $\mathrm{Z}=8.52(P<.001)$

Fig. 2. Effects of tiotropium versus placebo on peak expiratory flow (PEF). A. Change in morning PEF. B. Change in evening PEF.

(WMD $-0.18,95 \% \mathrm{CI}-0.36$ to $0.00 ; \mathrm{I}^{2}=0 \%, P=.06$ ), the difference was not statistically significant (Fig. 6).

Quality of Life. Three trials reported the AQLQ score. Although the cumulative analysis showed a little decrease in patients receiving tiotropium compared with those receiving placebo (WMD $0.09,95 \% \mathrm{CI}-0.01$ to 0.20 ; $\mathrm{I}^{2}=0 \%, P=.09$ ), no significant difference between the two groups was observed.

Adverse Events. The incidence of adverse events was evaluated in 6 studies. The overall cumulative incidence of adverse events was $44 \%$ in the tiotropium group and $47.4 \%$ in the placebo group. All the adverse events reported in at least 2 trials are shown in Table 3. The overall analysis showed no statistically significant increase in the total number of adverse events in the tiotropium group (OR 0.80, $95 \%$ CI $0.62-1.03, P=.08)$. Among adverse events, asthma exacerbations (OR $0.69,95 \%$ CI $0.54-0.89, P=.004$ ) and PEF rate decline decreased (OR 0.70, 95\% CI 0.52 $0.96, P=.02$ ) markedly in tiotropium group. There was no statistical significant difference in serious adverse events between the two groups (OR 1.15, 95\% CI $0.74-1.79$, $P=.54$ ) (Fig. 7).

\section{Discussion}

Asthma is a common airway obstructive diseases, and bronchodilators are very important to the management of asthma symptoms. ${ }^{21}$ The added benefits of combining two long-acting bronchodilators with different modes of action have been observed in patients with COPD. ${ }^{22}$ Tiotropium will be approved by the FDA for the treatment of asthma in the next few months. However, guidelines do not specifically recommend the addition of an inhaled long-acting anticholinergic drug to the current treatment of asthma. ${ }^{23}$ This meta-analysis incorporates 6 RCTs and includes data from 1,648 patients with inadequately controlled asthma. To our knowledge this is the first meta-analysis to date of the efficacy and safety of tiotropium versus placebo regarding clinically relevant outcomes in patients with inadequately controlled asthma who are receiving treatment with inhaled corticosteroids or inhaled corticosteroids plus long-acting $\beta_{2}$ agonists. The efficacy of tiotropium is evaluated by its impact on lung function and other clinical outcomes, including asthma control, quality of life, night awakenings, and rescue medication use.

This meta-analysis clearly shows the beneficial effects of the addition of tiotropium on lung function in inadequately controlled asthma patients who are receiving treat- 
A.

\begin{tabular}{|c|c|c|c|c|c|c|c|c|c|c|}
\hline \multirow{2}{*}{ Study or Subgroup } & \multicolumn{3}{|c|}{ Tiotropium } & \multicolumn{3}{|c|}{ Placebo } & \multirow{2}{*}{ Weight } & \multirow{2}{*}{$\begin{array}{l}\text { Mean Difference IV, } \\
\text { Random, 95\% CI }\end{array}$} & \multirow{2}{*}{\multicolumn{2}{|c|}{$\begin{array}{l}\text { Mean Difference IV, } \\
\text { Random, 95\% CI }\end{array}$}} \\
\hline & Mean & SD & Total & Mean & SD & Total & & & & \\
\hline Kerstjens 2012 trial-2 & 0.397 & 0.382 & 216 & 0.245 & 0.381 & 232 & $27.3 \%$ & $0.15[0.08,0.22]$ & & $\longrightarrow$ \\
\hline Kerstjens 2012-trial-1 & 0.367 & 0.4 & 237 & 0.295 & 0.387 & 222 & $26.7 \%$ & $0.07[-0.00,0.14]$ & & \\
\hline NCT01122680 & 0.602 & 0.404 & 77 & 0.489 & 0.404 & 74 & $10.4 \%$ & $0.11[-0.02,0.24]$ & & \\
\hline NCT01152450 & 0.468 & 0.427 & 90 & 0.337 & 0.427 & 90 & $11.0 \%$ & $0.13[0.01,0.26]$ & & \\
\hline NCT01233284 & 0.304 & 0.335 & 143 & 0.116 & 0.324 & 144 & $24.5 \%$ & $0.19[0.11,0.26]$ & & \\
\hline Total $(95 \%$ CI $)$ & & & 763 & & & 762 & $100.0 \%$ & $0.13[0.09,0.18]$ & & $n_{1}$ \\
\hline
\end{tabular}

Heterogeneity: $\mathrm{Tau}^{2}=0.00 ; \mathrm{Chi}^{2}=5.13, \mathrm{df}=4(P=.27) ; \mathrm{I}^{2}=22 \%$

Test for overall effect: $\mathrm{Z}=5.90(P<.001)$

B.

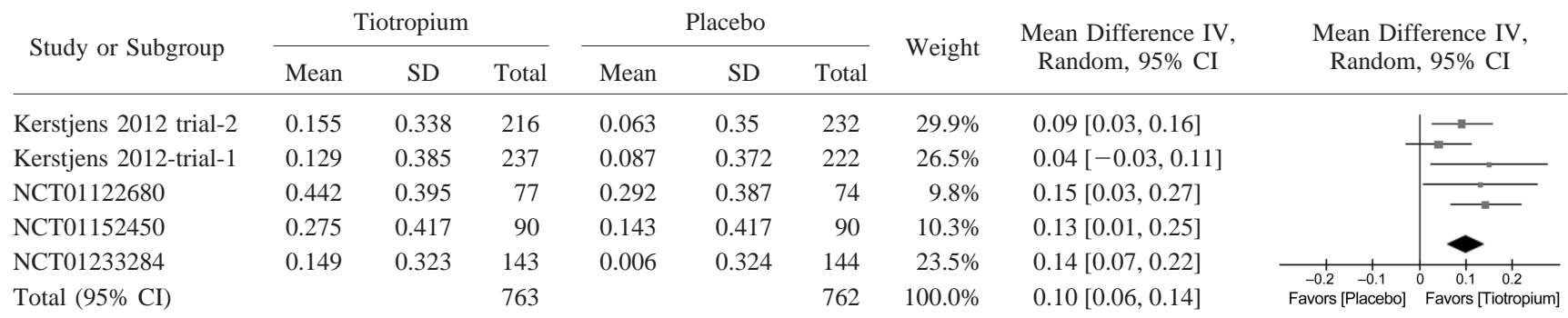

Heterogeneity: $\mathrm{Tau}^{2}=0.00 ; \mathrm{Chi}^{2}=4.90, \mathrm{df}=4(P=.30) ; \mathrm{I}^{2}=18 \%$

Test for overall effect: $\mathrm{Z}=4.80(P<.001)$

C.

\begin{tabular}{|c|c|c|c|c|c|c|c|c|c|c|}
\hline \multirow{2}{*}{ Study or Subgroup } & \multicolumn{3}{|c|}{ Tiotropium } & \multicolumn{3}{|c|}{ Placebo } & \multirow{2}{*}{ Weight } & \multirow{2}{*}{$\begin{array}{l}\text { Mean Difference IV, } \\
\text { Random, 95\% CI }\end{array}$} & \multirow{2}{*}{\multicolumn{2}{|c|}{$\begin{array}{l}\text { Mean Difference IV, } \\
\text { Random, 95\% CI }\end{array}$}} \\
\hline & Mean & SD & Total & Mean & SD & Total & & & & \\
\hline NCT01122680 & 0.497 & 0.395 & 77 & 0.363 & 0.387 & 74 & $12.2 \%$ & $0.13[0.01,0.26]$ & & \\
\hline NCT01233284 & 0.203 & 0.323 & 143 & 0.025 & 0.324 & 144 & $26.4 \%$ & $0.18[0.10,0.25]$ & & $\longrightarrow-$ \\
\hline Kerstjens 2012-trial-1 & 0.289 & 0.369 & 237 & 0.217 & 0.372 & 222 & $30.0 \%$ & $0.07[0.00,0.14]$ & & \\
\hline Kerstjens 2012 trial-2 & 0.31 & 0.353 & 216 & 0.172 & 0.35 & 232 & $31.5 \%$ & $0.14[0.07,0.20]$ & & 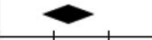 \\
\hline Total $(95 \% \mathrm{CI})$ & & & 673 & & & 672 & $100.0 \%$ & $0.13[0.08,0.18]$ & $\begin{array}{cc}-0.2 & -0.1 \\
\text { Favors [Placebo] }\end{array}$ & $\begin{array}{cc}0.1 & 0.2 \\
\text { Favors [Tiotropium] }\end{array}$ \\
\hline
\end{tabular}

Heterogeneity: $\mathrm{Tau}^{2}=0.00 ; \mathrm{Chi}^{2}=4.43, \mathrm{df}=3(P=.22) ; \mathrm{I}^{2}=32 \%$

Test for overall effect: $\mathrm{Z}=5.31(P<.001)$

Fig. 3. Effects of tiotropium versus placebo on $\mathrm{FEV}_{1}$. A. Change in peak FEV $\mathrm{F}_{1}$. B. Change in trough FEV $\mathrm{F}_{1}$. C. Change in area under the curve of the first $3 \mathrm{~h}$ of $\mathrm{FEV}_{1}$.

ment with inhaled corticosteroids or inhaled corticosteroids plus long-acting $\beta_{2}$ agonists. When compared with placebo, patients treated with tiotropium showed statistically significant improvements from baseline in all spirometric indices, including trough and peak $\mathrm{FEV}_{1}$, area under the curve of the first $3 \mathrm{~h}$ of $\mathrm{FEV}_{1}$, trough and peak FVC, area under the curve of the first $3 \mathrm{~h}$ of FVC, and morning and evening PEF. Although the improvement in $\mathrm{FEV}_{1}$ was not nearly the minimum clinically important difference of $230 \mathrm{~mL}$ in asthma, ${ }^{20}$ it should be noted that the increases were in patients who were receiving inhaled corticosteroids or inhaled corticosteroids plus long-acting $\beta_{2}$ agonists. There were no significant differences between tiotropium and placebo groups in AQLQ score, night awakenings, or use of rescue medication. Although a statisti- cally significant difference was reported for ACQ-7 score, it was not clinically important. This suggests that despite the good effects of tiotropium on lung function no significant effect on other clinical parameters was demonstrated. Only 2 studies by Kerstjens et al $^{16}$ reported data about exacerbations. Because the data currently available on exacerbations are insufficient for a meta-analysis, further investigation into the effects of tiotropium on exacerbations are required. Kerstjens et al $^{16}$ reported that the addition of tiotropium prolonged the time to the first severe exacerbation ( 282 vs 226 d) with an overall reduction of $21 \%$ in the risk of a severe exacerbation (hazard ratio 0.79 , $P=.03$ ). The results are inconsistent with a previous study. Peters et $\mathrm{al}^{11}$ found that the addition of tiotropium had no significant effect on asthma exacerbations although 
A.

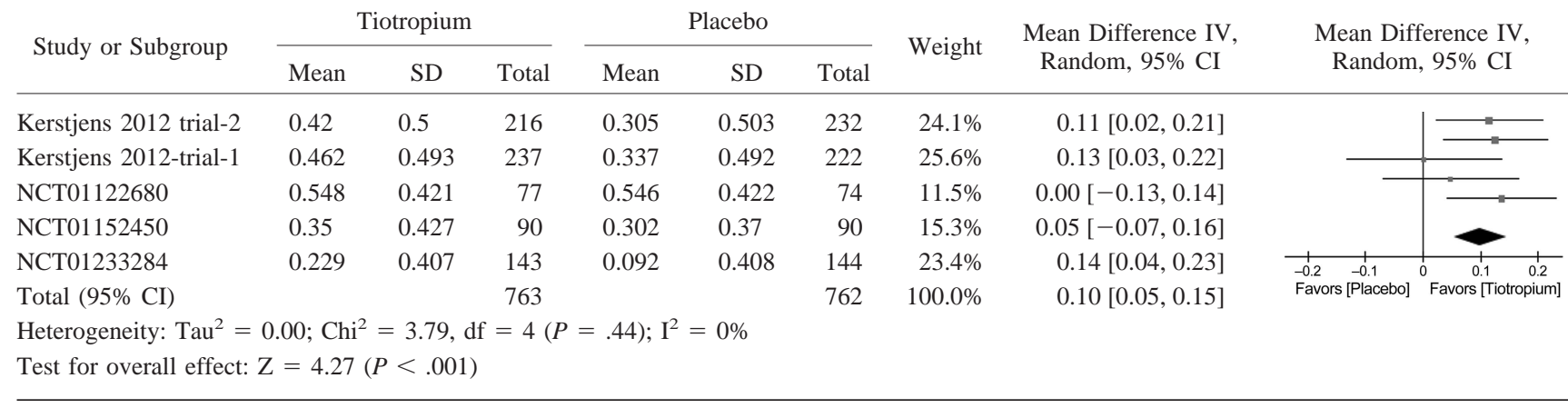

B.

\begin{tabular}{|c|c|c|c|c|c|c|c|c|c|c|}
\hline \multirow{2}{*}{ Study or Subgroup } & \multicolumn{3}{|c|}{ Tiotropium } & \multicolumn{3}{|c|}{ Placebo } & \multirow{2}{*}{ Weight } & \multirow{2}{*}{$\begin{array}{l}\text { Mean Difference IV, } \\
\text { Random, 95\% CI }\end{array}$} & \multirow{2}{*}{\multicolumn{2}{|c|}{$\begin{array}{l}\text { Mean Difference IV, } \\
\text { Random, 95\% CI }\end{array}$}} \\
\hline & Mean & SD & Total & Mean & SD & Total & & & & \\
\hline Kerstjens 2012 trial-2 & 0.142 & 0.47 & 216 & 0.072 & 0.472 & 232 & $26.8 \%$ & $0.07[-0.02,0.16]$ & & \\
\hline Kerstjens 2012-trial-1 & 0.173 & 0.477 & 237 & 0.062 & 0.477 & 222 & $26.8 \%$ & $0.11[0.02,0.20]$ & & \\
\hline NCT01122680 & 0.4 & 0.412 & 77 & 0.357 & 0.404 & 74 & $12.1 \%$ & $0.04[-0.09,0.17]$ & & \\
\hline NCT01152450 & 0.168 & 0.436 & 90 & 0.112 & 0.436 & 90 & $12.6 \%$ & $0.06[-0.07,0.18]$ & & \\
\hline NCT01233284 & 0.102 & 0.419 & 143 & 0.004 & 0.42 & 144 & $21.7 \%$ & $0.10[0.00,0.20]$ & $\begin{array}{cc}1 & 1 \\
-0.2 & -0.1\end{array}$ & \\
\hline Total $(95 \% \mathrm{CI})$ & & & 763 & & & 762 & $100.0 \%$ & $0.08[0.04,0.13]$ & Favors [Placebo] & Favors [Tiotropium] \\
\hline
\end{tabular}

Heterogeneity: $\mathrm{Tau}^{2}=0.00 ; \mathrm{Chi}^{2}=1.11, \mathrm{df}=4(P=.89) ; \mathrm{I}^{2}=0 \%$

Test for overall effect: $\mathrm{Z}=3.56(P=.004)$

C.

\begin{tabular}{|c|c|c|c|c|c|c|c|c|c|c|}
\hline \multirow{2}{*}{ Study or Subgroup } & \multicolumn{3}{|c|}{ Tiotropium } & \multicolumn{3}{|c|}{ Placebo } & \multirow{2}{*}{ Weight } & \multirow{2}{*}{$\begin{array}{l}\text { Mean Difference IV, } \\
\text { Random, 95\% CI }\end{array}$} & \multirow{2}{*}{\multicolumn{2}{|c|}{$\begin{array}{l}\text { Mean Difference IV, } \\
\text { Random, 95\% CI }\end{array}$}} \\
\hline & Mean & SD & Total & Mean & SD & Total & & & & \\
\hline Kerstjens 2012 trial-2 & 0.299 & 0.47 & 216 & 0.19 & 0.472 & 232 & $29.4 \%$ & $0.11[0.02,0.20]$ & & 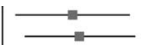 \\
\hline Kerstjens 2012-trial-1 & 0.344 & 0.462 & 237 & 0.223 & 0.462 & 222 & $31.3 \%$ & $0.12[0.04,0.21]$ & & \\
\hline NCT01122680 & 0.429 & 0.395 & 77 & 0.413 & 0.396 & 74 & $14.1 \%$ & $0.02[-0.11,0.14]$ & & \\
\hline NCT01233284 & 0.11 & 0.407 & 143 & -0.028 & 0.408 & 144 & $25.2 \%$ & $0.14[0.04,0.23]$ & & \\
\hline Total $(95 \% \mathrm{CI})$ & & & 673 & & & 672 & $100.0 \%$ & $0.11[0.06,0.15]$ & $\begin{array}{l}-0.2 \\
\text { Favors [Placebo] }\end{array}$ & $\begin{array}{l}0.1 \\
0 \\
0\end{array}$ \\
\hline
\end{tabular}

Heterogeneity: $\mathrm{Tau}^{2}=0.00 ; \mathrm{Chi}^{2}=2.52, \mathrm{df}=3(P=.47) ; \mathrm{I}^{2}=0 \%$

Test for overall effect: $\mathrm{Z}=4.43(P<.001)$

Fig. 4. Effects of tiotropium versus placebo on FVC. A. Change in peak FVC. B. Change in trough FVC. C. Change in area under the curve of the first $3 \mathrm{~h}$ of FVC.

\begin{tabular}{|c|c|c|c|c|c|c|c|c|c|}
\hline \multirow{2}{*}{ Study or Subgroup } & \multicolumn{3}{|c|}{ Tiotropium } & \multicolumn{3}{|c|}{ Placebo } & \multirow{2}{*}{ Weight } & \multirow{2}{*}{$\begin{array}{l}\text { Mean Difference IV, } \\
\text { Fixed, 95\% CI }\end{array}$} & \multirow{2}{*}{$\begin{array}{l}\text { Mean Difference IV, } \\
\text { Fixed, 95\% CI }\end{array}$} \\
\hline & Mean & SD & Total & Mean & SD & Total & & & \\
\hline NCT01122680 & -0.066 & 0.258 & 79 & -0.086 & 0.256 & 73 & $35.4 \%$ & $0.02[-0.06,0.10]$ & 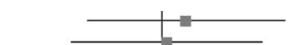 \\
\hline NCT01152450 & -0.102 & 0.272 & 88 & -0.106 & 0.267 & 91 & $37.9 \%$ & $0.00[-0.07,0.08]$ & - \\
\hline NCT01233284 & -0.187 & 0.408 & 144 & -0.156 & 0.405 & 142 & $26.7 \%$ & $-0.03[-0.13,0.06]$ & \\
\hline $\begin{array}{l}\text { Total }(95 \% \mathrm{CI}) \\
\text { Heterogeneity: } \mathrm{Chi}^{2}=\end{array}$ & $5, \mathrm{df}=$ & $=$ & $\begin{array}{l}311 \\
; \mathrm{I}^{2}=\end{array}$ & & & 306 & $100.0 \%$ & $0.00[-0.05,0.05]$ & $\begin{array}{ccccc}1 & 1 & 1 \\
-0.1 & -0.05 & 0 & 0.05 & 0.1 \\
\text { Favors [Tiotropium] } & \text { Favors [Placebo] }\end{array}$ \\
\hline
\end{tabular}

Test for overall effect: $\mathrm{Z}=0.01(P=.99)$

Fig. 5. Effects of tiotropium versus placebo on night awakenings.

a trend was observed toward a better effect of tiotropium compared with a higher dose of inhaled corticosteroid. The difference may be attributed to the treatment course in the study by Peters et al, ${ }^{11}$ which was too short to examine the rate of asthma exacerbations. Moreover, the study by Peters et al ${ }^{11}$ compared tiotropium with salmeterol and a higher 


\begin{tabular}{|c|c|c|c|c|c|c|c|c|c|}
\hline \multirow{2}{*}{ Study or Subgroup } & \multicolumn{3}{|c|}{ Tiotropium } & \multicolumn{3}{|c|}{ Placebo } & \multirow{2}{*}{ Weight } & \multirow{2}{*}{$\begin{array}{l}\text { Mean Difference IV, } \\
\text { Fixed, 95\% CI }\end{array}$} & \multirow{2}{*}{$\begin{array}{l}\text { Mean Difference IV, } \\
\text { Fixed, 95\% CI }\end{array}$} \\
\hline & Mean & $\mathrm{SD}$ & Total & Mean & SD & Total & & & \\
\hline Kerstjens 2012 trial-2 & -1.144 & 2.396 & 216 & -0.881 & 2.407 & 232 & $16.9 \%$ & $-0.26[-0.71,0.18]$ & 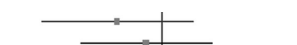 \\
\hline Kerstjens 2012-trial-1 & -0.806 & 2.078 & 237 & -0.714 & 2.086 & 222 & $23.0 \%$ & $-0.09[-0.47,0.29]$ & \\
\hline NCT01122680 & -0.528 & 1.342 & 79 & -0.412 & 1.324 & 73 & $18.6 \%$ & $-0.12[-0.54,0.31]$ & - \\
\hline NCT01152450 & -1.106 & 1.792 & 89 & -0.841 & 1.803 & 91 & $12.1 \%$ & $-0.27[-0.79,0.26]$ & \\
\hline $\begin{array}{l}\text { NCT01233284 } \\
\text { Total }(95 \% \text { CI) }\end{array}$ & -0.769 & 1.464 & $\begin{array}{l}144 \\
765\end{array}$ & -0.569 & 1.454 & $\begin{array}{l}142 \\
760\end{array}$ & $\begin{array}{r}29.3 \% \\
100.0 \%\end{array}$ & $\begin{array}{l}-0.20[-0.54,0.14] \\
-0.18[-0.36,0.00]\end{array}$ & 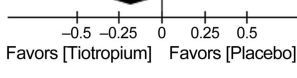 \\
\hline
\end{tabular}

Heterogeneity: $\mathrm{Chi}^{2}=0.54, \mathrm{df}=4(P=.97) ; \mathrm{I}^{2}=0 \%$

Test for overall effect: $\mathrm{Z}=1.91(P=.06)$

Fig. 6. Effects of tiotropium versus placebo on rescue medication use.

Table 3. Adverse Events With Tiotropium Compared With Placebo

\begin{tabular}{|c|c|c|c|c|c|c|c|}
\hline Events & Trials & Participants, $n$ & Tiotropium, $\%$ & Placebo, $\%$ & OR & $95 \% \mathrm{CI}$ & $P$ \\
\hline \multicolumn{8}{|l|}{ Serious adverse events } \\
\hline Gastrointestinal disorders & 4 & 1,456 & 0.685 & 0.551 & 1.19 & $0.36-3.90$ & .78 \\
\hline General disorders & 2 & 912 & 0.219 & 0.219 & 1 & $0.14-7.24$ & $>.99$ \\
\hline Infections and infestations & 3 & 1,166 & 1.370 & 1.031 & 1.35 & $0.48-3.78$ & .57 \\
\hline Injury & 3 & 1,094 & 0.733 & 0.548 & 1.29 & $0.32-5.20$ & .72 \\
\hline Musculoskeletal and connective tissue disorders & 2 & 912 & 0.658 & 1.096 & 0.67 & $0.18-2.41$ & .54 \\
\hline $\begin{array}{l}\text { Neoplasms benign, malignant,and unspecified } \\
\text { (including cysts and polyps) }\end{array}$ & 2 & 912 & 0.877 & 0.439 & 1.87 & $0.39-8.86$ & .43 \\
\hline Nervous system disorders & 3 & 1,067 & 0.560 & 0.377 & 1.33 & $0.29-6.03$ & .71 \\
\hline Psychiatric disorders & 2 & 743 & 1.096 & 0 & 5.19 & $0.60-44.67$ & .13 \\
\hline Respiratory, thoracic, and mediastinal disorders & 2 & 912 & 4.825 & 4.825 & 1 & $0.55-1.83$ & $>.99$ \\
\hline Vascular disorders & 2 & 635 & 1.294 & 0.613 & 2.11 & $0.38-11.61$ & .39 \\
\hline \multicolumn{8}{|l|}{ Other adverse events } \\
\hline Infections and infestations & 3 & 1,166 & 18.322 & 17.698 & 1.06 & $0.78-1.44$ & .69 \\
\hline PEF rate decreased & 2 & 912 & 20.395 & 26.754 & 0.7 & $0.52-0.96$ & .02 \\
\hline Nervous system disorders & 3 & 1,094 & 5.495 & 6.022 & 0.92 & $0.55-1.53$ & .74 \\
\hline Asthma & 3 & 1,166 & 30.993 & 39.003 & 0.69 & $0.54-0.89$ & .004 \\
\hline $\begin{array}{l}\mathrm{PEF}=\text { peak expiratory flow } \\
\mathrm{OR}=\text { odds ratio }\end{array}$ & & & & & & & \\
\hline
\end{tabular}

dose of inhaled corticosteroid while that by Kerstjens et al ${ }^{16}$ compared tiotropium with placebo in patients with poorly controlled asthma. Hospitalization and severe asthma exacerbations will affect the quality of life in subjects with asthma. Although Kerstjens et al ${ }^{16}$ found a significantly longer time to the first exacerbation, our results showed no significant differences between the tiotropium and placebo groups in AQLQ score. The AQLQ was developed to measure patients' functional experiences over a 2-week period, and it asks patients to recall their experiences during the previous days. Therefore, it is not suitable for capturing the rapidly changing experiences that occur during an acute asthma exacerbation..$^{30}$ Furthermore, although the difference was not statistically significant, it was noticeable that there were trends toward the improvement of
AQLQ score $(P=.09)$ and a reduction in the number of instances of rescue medication use $(P=.06)$ among patients treated with tiotropium. It indicated that the increase in sample size might get a positive result.

It was surprising for us to find a decreasing although statistically insignificant trend in this meta-analysis in the total number of adverse events among patients treated with tiotropium. Among all adverse events, there was an obvious decrease in asthma exacerbations and PEF rate decline in the tiotropium group, which might account for the decreasing trend in total number of adverse events among patients treated with tiotropium. In addition, no significant increase in serious adverse events was observed. Dry mouth, urinary retention, and cardiovascular events are the most concerning adverse side effects of anticholinergic agents. 


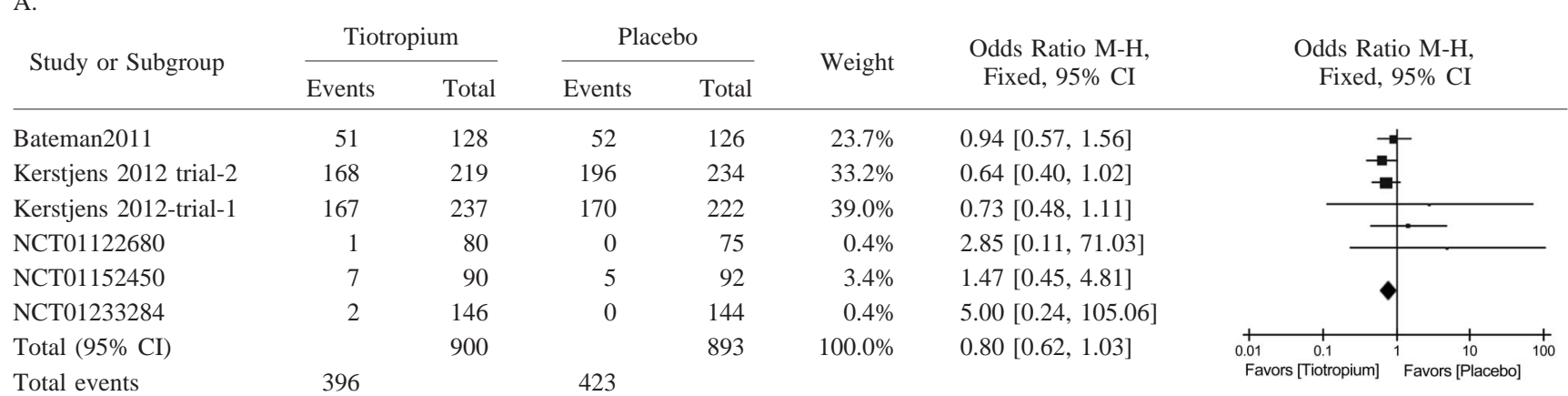

Heterogeneity: $\mathrm{Chi}^{2}=4.48, \mathrm{df}=5(P=.48) ; \mathrm{I}^{2}=0 \%$

Test for overall effect: $\mathrm{Z}=1.73(P=.08)$

B.



Heterogeneity: $\mathrm{Chi}^{2}=3.30$, df $=5(P=65) ; \mathrm{I}^{2}=0 \%$

Test for overall effect: $\mathrm{Z}=0.61(P=.54)$

Fig. 7. Effects of tiotropium versus placebo on adverse events. A. Total adverse events. B. Serious adverse events.

This analysis showed that these adverse events, which were of mild-to-moderate severity according to statements in the relevant articles, were reported in a very small portion of the patients included in the study. It should be noted that the low incidence of cardiovascular events might have resulted from the exclusion of patients with serious cardiovascular diseases in the trials included for this metaanalysis. Excess cardiovascular events might have been anticipated in such patients.

We are very interested in the studies on tiotropium that used a powder inhaler in the treatment of asthma, because Handihaler is the only device now available in China for administering tiotropium. We found 6 RCTs on tiotropium that used the powder inhaler in an asthma population. Of the 6 RCTs, one evaluated the addition of tiotropium to an inhaled glucocorticoid, compared with a doubling of the inhaled glucocorticoid or the addition of salmeterol. ${ }^{11}$ The results showed that tiotropium improved symptoms and lung function in patients with inadequately controlled asthma when added to an inhaled glucocorticoid. Its effects appeared to be equivalent to those with the addition of salmeterol. To reduce heterogeneity of different trials, we selected only the data comparing tiotropium with pla- cebo. Another RCT was designed to determine the spirometric effects of tiotropium in COPD patients with concomitant asthma. ${ }^{24}$ The results showed that the patients with COPD and concomitant asthma achieved spirometric improvements with tiotropium treatment along with symptomatic benefits as seen by a reduced need for rescue medication. To reduce the heterogeneity of different trials, we also selected only data from asthma patients without other lung diseases. An RCT by Fardon et $\mathrm{al}^{25}$ compared tiotropium with placebo, but the data it provided were not suitable for our meta-analysis. Three RCTs investigated the protection of tiotropium administered with a powder inhaler device versus that of placebo or other anticholinergic drugs against methacholine-induced bronchoconstriction in patients with asthma, ${ }^{26-28}$ but they were not relevant to this meta-analysis. Hence, we excluded the 6 RCTs on tiotropium that used a powder inhaler before ultimate analysis.

The main strength of our study was the inclusion of a large pool of patients with inadequately controlled asthma, allowing us to perform robust analysis of clinically relevant outcomes following the addition of tiotropium versus placebo to standard treatment strategy. The trials included in this analysis were of good quality and used almost 
identical designs with regard to inclusion and exclusion criteria. And the clinical characteristics of study populations were quite homogeneous. However, the results should be interpreted with caution because they might have been influenced by other factors. First, there were differences in trial duration. The duration of treatment in most trials here was too short to allow adequate evaluation of the longterm efficacy and safety of tiotropium. Although a metaanalysis showed a $46 \%$ relative risk increase of death in COPD trials that used the $5 \mu \mathrm{g}$ tiotropium Respimat inhaler, ${ }^{29}$ it has not been elucidated whether the increase in death among asthma patients was brought about by the use of the Respimat inhaler. Further long-term studies are anticipated to answer this question. Second, the patients with inadequately controlled asthma included in this meta-analysis were $\geq 12$ y old, free from other pulmonary diseases, and in non-smoking status. Therefore, it is inappropriate to generalize the results of this meta-analysis to all asthma patients. Third, the trials included in this analysis had different criteria for the use of comedications. In the current trials, tiotropium is a medicine to be added to standard treatment regimens rather than a first-choice medicine.

The clinical homogeneity of the trials resulted in statistical homogeneity for all outcome measures across the trials. Selection bias was avoided using a systematic search strategy, and we specified the inclusion and exclusion criteria. Furthermore, two reviewers independently evaluated the selected studies, and a third reviewer was consulted to reach consensus if necessary. Double counting of patients from overlapping publications was avoided. Funnel plots for the primary end point showed no clear evidence of publication bias. Selective reporting of secondary end points and non-intention-to-treat reports in published manuscripts may have biased the results. We minimized this bias by obtaining supplemental data for the included studies.

\section{Conclusions}

This meta-analysis indicates that the addition of tiotropium to the treatment of inadequately controlled asthma, compared with placebo, may improve lung function and not increase the number of adverse events. Because of the limitations of this meta-analysis, we suggest that further work should be required to compare the addition of tiotropium with that of placebo. Larger, longer, multicenter, double-blind, parallel RCTs are expected to validate the efficacy and safety of the addition of tiotropium to standard treatment regimens for inadequately controlled asthma.

\section{ACKNOWLEDGMENTS}

We thank Guang-qiao Zeng MD, State Key Laboratory of Respiratory Disease, Guangzhou Medical University, Guangzhou, China, for his assistance in medical writing and statistical advice.

\section{REFERENCES}

1. Fanta CH. Asthma. N Engl J Med 2009;360(10):1002-1014.

2. Global Initiative for Asthma (GINA): Global Strategy for Asthma Management and Prevention. Updated 2012. http://www.ginasthma. org. Accessed May 12, 2013.

3. Matera MG, Cazzola M. Ultra-long-acting beta2-adrenoceptor agonists: an Emerging therapeutic option for asthma and COPD? Drugs 2007;67(4):503-515.

4. Tashkin DP, Celli B, Senn S, Burkhart D, Kesten S, Menjoge S, et al. A 4-year trial of tiotropium in chronic obstructive pulmonary disease. N Engl J Med 2008;359(15):1543-1554.

5. Westby M, Benson M, Gibson P. Anticholinergic agents for chronic asthma in adults. Cochrane Database Syst Rev 2004;3:CD003269.

6. Casale TB, Eucklund P. Characterization of muscarinic receptor subtypes on human peripheral lung. J Appl Physiol 1988;65(2):594600.

7. Barnes PJ. Muscarinic receptor subtypes in airways. Life Sci 1993; 52(5-6):521-527.

8. Global Initiative for Chronic Obstructive Lung Disease (GOLD). Report of the GOLD Workshop: Global Strategy for Diagnosis, Management and Prevention of COPD. Updated 2012. http://www.goldcopd.com. Accessed May 12, 2013.

9. Ohta S, Oda N, Yokoe T, Tanaka A, Yamamoto Y, Watanabe Y, et al. Effect of tiotropium bromide on airway inflammation and remodeling in a mouse model of asthma. Clin Exp Allergy 2010;40(8):1266-1275.

10. Buels KS, Jacoby DB, Fryer AD. Non-bronchodilating mechanisms of tiotropium prevent airway hyperreactivity in a guinea-pig model of allergic asthma. Br J Pharmacol 2012;165(5):1501-1514.

11. Peters SP, Kunselman SJ, Icitovic N, Moore WC, Pascual R, Ameredes BT, et al. Tiotropium bromide step-up therapy for adults with poorly controlled asthma. N Engl J Med 2010;363(18):1715-1726.

12. Bateman ED, Kornmann O, Schmidt P, Pivovarova A, Engel M, Fabbri LM. Tiotropium is noninferior to salmeterol in maintaining improved lung function in B16 Arg/Arg patients with asthma. J Allergy Clin Immunol 2011;128(2):315-322.

13. Jadad AR, Moore RA, Carroll D, Jenkinson C, Reynolds DJ, Gavaghan DJ, et al. Assessing the quality of reports of randomized clinical trials: is blinding necessary? Control Clin Trials 1996;17(1):1-12.

14. DerSimonian R, Laird N. Meta-analysis in clinical trials. Control Clin Trials 1986;7(3):177-188.

15. Egger M, Smith GD. Bias in location and selection of studies. BMJ 1998;316(7124):61-66.

16. Kerstjens HA, Disse B, Schroder-Babo W, Bantje TA, Gahlemann M, Sigmund R, et al. Tiotropium improves lung function in patients with severe poorly controlled asthma: a randomized controlled trial. J Allergy Clin Immunol 2011;128(2):308-314.

17. Efficacy and safety of 3 doses of tiotropium compared to placebo in adolescents (12 to 17 Yrs) with moderate asthma. Updated 2012. http:// clinicaltrial.gov/ct2/show/NCT01122680. Accessed May 12, 2013.

18. Randomised, double-blind, cross-over efficacy and safety comparison of three different doses of tiotropium administered once daily versus placebo in patients with moderate persistent asthma. http:// clinicaltrial.gov/ct2/show/NCT01152450. Accessed May 12, 2013.

19. A randomised, double-blind, placebo controlled, cross-over efficacy and safety comparison of tiotropium $5 \mu \mathrm{g}$ once daily and tiotropium $2.5 \mu \mathrm{g}$ twice daily for four weeks in patients with moderate persistent asthma. http://clinicaltrial.gov/ct2/show/NCT01233284. Accessed May 12, 2013.

20. Santanello NC, Zhang J, Seidenberg B, Reiss TF, Barber BL. What are minimal important changes for asthma measures in a clinical trial? Eur Respir J 1999;14(1):23-27.

21. National Institutes of Health, National Heart, Lung and Blood Institute. Global initiative for asthma; global strategy for asthma man- 
agement and prevention. National Institutes of Health, Publication 02-3659, 2002.

22. van Noord JA, Aumann JL, Janssens E, Smeets JJ, VVerhaert J, Disse B, et al. Comparison of tiotropium once daily, formoterol twice daily and both combined once daily in patients with COPD. Eur Respir J 2005;26(2):214-222.

23. Bateman ED, Boushey HA, Bousquet J, Busse WW, Clark TJ, Pauwels RA, et al. Can guideline-defined asthma control be achieved? The Gaining Optimal Asthma Control Study. Am J Respir Crit Care Med 2004;170(8):836-844.

24. Magnussen H, Bugnas B, van Noord J, Schmidt P, Gerken F, Kesten S. Improvements with tiotropium in COPD patients with concomitant asthma. Respir Med 2008;102(1):50-56.

25. Fardon T, Haggart K, Lee DK, Lipworth BJ. A proof of concept study to evaluate stepping down the dose of fluticasone in combination with salmeterol and tiotropium in severe persistent asthma. Respir Med 2007;101(6):1218-1228.
26. O'Connor BJ, Towse LJ, Barnes PJ. Prolonged effect of tiotropium bromide on methacholine-induced bronchoconstriction in asthma. Am J Respir Crit Care Med 1996;154(4):876-880.

27. Sposato B, Barzan R, Calabrese A, Franco C. Comparison of the protective effect amongst anticholinergic drugs on methacholineinduced bronchoconstriction in asthma. J Asthma 2008;45(5):397401.

28. Terzano C, Petroianni A, Ricci A, D’Antoni L, Allegra L. Early protective effects of tiotropium bromide in patients with airways hyperresponsiveness. Eur Rev Med Pharmacol Sci 2004;8(6):259-264.

29. Singh S, Loke YK, Enright PL, Furberg CD. Mortality associated with tiotropium mist inhaler in patients with chronic obstructive pulmonary disease: systematic review and meta-analysis of randomised controlled trials. BMJ 2011;342:d3215.

30. Juniper EF, Svensson K, Mörk AC, Ståhl E. Measuring health-related quality of life in adults during an acute asthma exacerbation. Chest 2004;125(1):93-97.

This article is approved for Continuing Respiratory Care Education credit. For information and to obtain your CRCE

(free to AARC members) visit

www.rcjournal.com 